\title{
Efficacia e sicurezza del trattamento con Setmelanotide, un agonista del recettore melanocortinico 4 , in pazienti con obesità grave causata da mutazioni dei geni LEPR, PCSK1 e POMC
}

\author{
Donatella Gilio $^{1}$ - Giovanni Ceccarini ${ }^{1}$
}

Accettato: 22 febbraio 2021 / Pubblicato online: 16 agosto 2021

(c) The Author(s) 2021

Commento a:

Efficacy and safety of Setmelanotide, an MC4R agonist, in individuals with severe obesity due to LEPR or POMC deficiency: single-arm, open-label, multicenter, phase 3 trials.

K. Clément, E. van den Akker, J. Argente, A. Bahm,

W.K. Chung, H. Connors, K. De Waele,

I. Sadaf Farooqi, J. Gonneau-Lejeune, G. Gordon,

K. Kohlsdorf, C. Poitou, L. Puder, J. Swain, M. Stewart,

G. Yuan, M. Wabitsch, P. Kühnen, Setmelanotide

POMC and LEPR Phase 3 Trial Investigators.

Lancet Diabetes Endocrinol (2020) 8(12):960-970

L'obesità è una malattia cronica caratterizzata da un eccesso di tessuto adiposo che riduce l'aspettativa di vita e determina un' aumentata morbilità e mortalità [1]. Circa il 2-5\% dei casi di obesità grave e a insorgenza precoce sono causati da alterazioni monogeniche che riducono la funzione della via di segnale della melanocortina, uno dei principali meccanismi di regolazione del peso corporeo.

La leptina, un ormone prodotto dal tessuto adiposo sottocutaneo, si lega al suo recettore (LEPR) espresso, tra gli altri, sulla membrana dei neuroni ipotalamici produttori di proopiomelanocortina (POMC); tale legame stimola la sintesi di POMC la quale viene poi clivata dall'enzima proproteina convertasi subtilisina e kexina tipo 1 (PCSK1) in vari neuro-peptidi. Uno di questi neuro-peptidi è l' $\alpha$-MSH che, attivando il recettore melanocortinico 4 (MC4R), induce un senso di sazietà con conseguente riduzione dell'assunzione

G. Ceccarini

giovanni.ceccarini@unipi.it

1 Centro Obesità e Lipodistrofie, Unità Operativa di Endocinologia I, Dipartimento di Medicina Clinica e Sperimentale, Azienda Ospedaliero-Universitaria, Pisa, Italia di cibo [2]. Le varianti bialleliche dei geni POMC, PCSK1 e LEPR, che riducono l'attività biologica dei loro prodotti, causano iperfagia cronica che determina l'insorgenza di forme di obesità grave, precoce [3] e particolarmente refrattarie alle terapie tradizionali. Il Setmelanotide è un analogo dell' $\alpha$-MSH e agonista di MC4R già dimostrato efficace nella riduzione del peso corporeo nei pazienti che presentano una ridotta produzione del potente anoressizzante $\alpha$-MSH [4].

In questo studio multicentrico, aperto, a singolo braccio, di fase 3, gli autori hanno valutato l'efficacia e la sicurezza della terapia con Setmelanotide in pazienti affetti da obesità secondaria a un deficit di LEPR o di POMC. Sono stati arruolati 10 pazienti con deficit di POMC (omozigosi o eterozigosi composta di POMC o PCSK1) e 11 pazienti con difetto di LEPR (omozigosi o eterozigosi composta del gene LEPR) che rispettavano i seguenti criteri: BMI $\geq 30 \mathrm{Kg} / \mathrm{m}^{2}$ per età $\geq 18$ anni o $\mathrm{BMI} \geq 95^{\circ}$ percentile per le età comprese fra i 6 e i 18 anni. Tutti i pazienti arruolati hanno ricevuto una dose terapeutica individualizzata per 12 settimane; coloro che alla fine di questo periodo hanno raggiunto un calo ponderale di almeno $5 \mathrm{Kg}$ (o pari al $5 \%$ se il peso iniziale era inferiore a $100 \mathrm{Kg}$ ) hanno preso parte alla fase successiva dello studio. In tale fase, i partecipanti hanno ricevuto il farmaco (in doppio cieco) per le prime 4 settimane e il placebo per le successive 4; in seguito, tutti i pazienti hanno ripreso la terapia con Setmelanotide alla dose precedentemente stabilita per altre 32 settimane.

Scopo principale di questo studio è stato quello di valutare la percentuale di pazienti che hanno ottenuto un calo ponderale di almeno il $10 \%$ rispetto al peso iniziale, a distanza di circa 1 anno dall'inizio della terapia. Si è inoltre quantificato, sempre a distanza di circa 12 mesi, il cambiamento nella sensazione di fame. I risultati hanno evidenziato come l' $80 \%$ dei pazienti con deficit di POMC o PCSK1 e il 
45\% di quelli con LEPR hanno raggiunto l'obiettivo primario, ovvero un calo ponderale di almeno il $10 \%$ rispetto al peso iniziale. Inoltre, si è registrata una riduzione dello score relativo alla sensazione di fame di circa il $27 \%$ nei pazienti arruolati nello studio POMC e di circa il $43 \%$ in quelli dello studio LEPR. Nei soggetti trattati con Setmelanotide non si sono verificati eventi avversi gravi, confermandone la sicurezza di impiego. Gli effetti collaterali più frequenti risultavano: reazione locale a livello della zona di iniezione, iperpigmentazione cutanea e nausea.

Questi risultati molto incoraggianti sono un esempio di applicazione del concetto di medicina di precisone e hanno portato alla recente approvazione da parte di FDA di Setmelanotide per pazienti di età superiore ai 6 anni e affetti da deficit di POMC PCSK1 o LEPR confermati geneticamente.

Saranno importanti ulteriori studi per verificare il potenziale impiego di Setmelanotide nel contesto di forme sindromiche di obesità (quali ad esempio le sindromi di BardetBiedl e Alström) e per verificarne l'efficacia nella pratica clinica quotidiana.

Funding Note Open access funding provided by Università di Pisa within the CRUI-CARE Agreement.

Nota della casa editrice Springer Nature rimane neutrale in riguardo alle rivendicazioni giurisdizionali nelle mappe pubblicate e nelle affiliazioni istituzionali.
Open Access This article is licensed under a Creative Commons Attribution 4.0 International License, which permits use, sharing, adaptation, distribution and reproduction in any medium or format, as long as you give appropriate credit to the original author(s) and the source, provide a link to the Creative Commons licence, and indicate if changes were made. The images or other third party material in this article are included in the article's Creative Commons licence, unless indicated otherwise in a credit line to the material. If material is not included in the article's Creative Commons licence and your intended use is not permitted by statutory regulation or exceeds the permitted use, you will need to obtain permission directly from the copyright holder. To view a copy of this licence, visit http://creativecommons.org/licenses/by/4.0/.

\section{Bibliografia}

1. Afshin A, Forouzanfar MH, Reitsma MB et al (2017) Health effects of overweight and obesity in 195 countries over 25 years. N Engl J Med 377:13-27

2. Cone RD (2005) Anatomy and regulation of the central melanocortin system. Nat Neurosci 5:571-578

3. Huszar D, Lynch CA, Fairchild-Huntress V et al (1997) Targeted disruption of the melanocortin-4 receptor results in obesity in mice. Cell 88:131-141

4. Kühnen P, Clément K, Wiegand S et al (2016) Proopiomelanocortin deficiency treated with a melanocortin-4 receptor agonist. N Engl J Med 375:240-246 